\title{
Evolution of Kerr-Schild type initial data for binary black holes using the horizon penetrating Teukolsky equation
}

\author{
Gaurav Khanna \\ Natural Science Division, \\ Southampton College of Long Island University, \\ Southampton NY 11968.
}

\begin{abstract}
We use the Kerr-Schild type Teukolsky equation (horizon penetrating) to evolve binary black hole initial data as proposed by Bishop et al. in the close limit. Our results are in agreement with those recently obtained by Sarbach et al. from the Zerilli equation evolution of the same initial data.
\end{abstract}

\section{INTRODUCTION}

There is a lot of current interest in studying the collision of two black holes since these events could be primary sources of gravitational waves for interferometric gravitational wave detectors currently under construction. Numerical simulations are considered as the key to model such collisions. Unfortunately, current computer limitations cause these simulations to be short lived, so to study the actual merger of two holes, one needs to have initial data that represents two black holes close to each other.

Early attempts to provide binary black hole initial data solved the initial value problem of General Relativity using conformally flat slices [1]. This restriction was too strong; it was unable to incorporate spinning holes. Lately, some effort has been geared towards obtaining initial data based on the Kerr-Schild form of black hole solutions. The main advantage of this form is that the spatial slices of these solutions are horizon penetrating, which make them ideal for the application of the "excision" method [6] for evolving black holes. This form of the solution also naturally incorporates boosted and spinning black holes.

There is also another way [5] to obtain initial data for a black hole binary, without solving the initial value equations directly. One could, in principle, consider a scenario with matter fields coupled to gravity such that there are no black holes to begin with. One could distribute the matter fields in such a way that their gravitational effects are like those of two stars that are separated far apart but each is critically close to gravitational collapse. It is quite possible that such a simulation would not be plagued with the subtle problems that ocurr when one attempts to evolve two black holes. One could set the parameters of the simulation in such a way that when the two stars come close to each other, they collapse upon themselves and form two black holes. This final slice could be used as initial data for a binary black hole code.

However in this paper, we consider the Bishop et al. 2] family of initial data wherein two non spinning Kerr-Schild black holes are superposed. This proposal has the property that in the "close limit" in which the separation of the holes is small, the metric is given by a distorted Kerr-Schild black hole. The evolution will be carried out using a recently introduced horizon penetrating Teukolsky formalism [7]. We present Teukolsky waveforms from this evolution and the amount of energy radiated away by the system. The results we obtain are in agreement with those obtained by Sarbach et al. [3] from their evolution of the same initial data family using a Kerr-Schild type Zerilli equation.

\section{KERR-SCHILD INITIAL DATA}

For the sake of completion, in the first subsection of this section we reproduce the calculations followed by Sarbach et al. 33] for an exact solution of the linearized initial value problem.

The KS space-time metric is defined by

$$
g_{\mu \nu}=\eta_{\mu \nu}-2 V k_{\mu} k_{\nu},
$$

where $k_{\mu}$ is a null vector. The $\eta_{\mu \nu}$ is taken to be the Minkowski metric with coordinates $(t, \underline{x})=\left(t, x^{i}\right)$ such that $\eta_{t t}=-1, \eta_{t i}=0$ and $\eta_{i j}=\delta_{i j}$. The null vector $k_{\mu}$ satisfies $k_{t}=-1$ and $k^{i} k_{i}=1$, where $k^{i}=\delta^{i j} k_{j}$. The three metric and extrinsic curvature with respect to a slice $t=$ const. are

$$
\begin{aligned}
\bar{g}_{i j} & =\delta_{i j}-2 V k_{i} k_{j}, \\
K_{i j} & =-\frac{1}{\alpha} \partial_{t}\left(V k_{i} k_{j}\right)+2 \alpha\left[V k^{s} \nabla_{s}\left(V k_{i} k_{j}\right)-\nabla_{(i}\left(V k_{j}\right)\right],
\end{aligned}
$$

where $\alpha=(1-2 V)^{-1 / 2}$ is the lapse and where $\nabla$ refers to the flat metric $\delta_{i j}$. 
Bishop et al.'s solution procedure [2] consists in inserting equations 1 and 2 into the constraint equations and to solve the resulting equations for $V, \dot{V}=\partial_{t} V$ and $\dot{k}_{i}=\partial_{t} k_{i}$, where $k_{i}$ is assumed to be given.

\section{A. Two black hole data}

Here we review the discussion of equations 11 and 2 as followed by Sarbach et al. where a particular ansatz is made for $k_{i}$ representing two nearby non-rotating and non-spinning black holes. A single Schwarzschild black hole can be written as

$$
k_{i}=\frac{\nabla_{i} \phi}{|\nabla \phi|}, \quad|\nabla \phi|^{2}=\delta^{i j} \nabla_{i} \phi \cdot \nabla_{j} \phi,
$$

with $\phi=1 / r$. For two black holes, Bishop et al. choose

$$
\phi(\underline{x})=\frac{M_{1}}{\left|\underline{x}-\underline{x}_{1}\right|}+\frac{M_{2}}{\left|\underline{x}-\underline{x}_{2}\right|},
$$

where $\underline{x}_{1}$ and $\underline{x}_{2}$ are the positions of the black holes that have mass $M_{1}$ and $M_{2}$. If the black holes are located at $\underline{x}_{1}=\left(0,0, a_{1}\right)$ and $\underline{x}_{2}=\left(0,0, a_{2}\right)$, with $a_{1}>0>a_{2}, \phi$ may be expanded in a sum over multipoles:

$$
\phi(r, \vartheta)=\sum_{\ell=0}^{\infty} \frac{M_{1} a_{1}^{\ell}+M_{2} a_{2}^{\ell}}{r^{\ell+1}} P_{\ell}(\cos \vartheta),
$$

where $P_{\ell}$ denote standard Legendre polynomials and where $(r, \vartheta, \varphi)$ are polar coordinates for $\underline{x}$. Note that the expansion (3) is only valid for $r>\max \left\{a_{1},-a_{2}\right\}$. We define the separation parameter as

$$
\varepsilon=\frac{a_{1}-a_{2}}{M},
$$

where $M=M_{1}+M_{2}$ is the total mass. Now, using the center of mass condition $M_{1} a_{1}+M_{2} a_{2}=0$, the close limit of (3) becomes

$$
\phi=\frac{M}{r}+\varepsilon^{2} \frac{M M_{1} M_{2}}{r^{3}} P_{2}(\cos \vartheta)+\mathcal{O}\left(\varepsilon^{3} / r^{4}\right) .
$$

As a result, to first order in $\varepsilon^{2}, k_{i}$ is given by

$$
k_{r}=-1, \quad k_{A}=\varepsilon^{2} \frac{M_{1} M_{2}}{r} \hat{\nabla}_{A} P_{2},
$$

where here and in the following, $A=\vartheta, \varphi$. The remaining amplitudes are expanded according to

$$
V=-\frac{M}{r}+\varepsilon^{2} v(r) P_{2}, \quad \dot{V}=\varepsilon^{2} \dot{v}(r) P_{2}, \quad \dot{k}_{A}=\varepsilon^{2} \dot{k}(r) \hat{\nabla}_{A} P_{2} .
$$

Plugging this into the constraint equations, and keeping only linear terms (of the order $\varepsilon^{2}$ ), one obtains the equations

$$
\begin{aligned}
& 0=-\dot{v}+\frac{3 M}{r^{2}}\left(1+\frac{2 M}{r}\right) \dot{k}-\frac{3}{r} v-\frac{6 M M_{1} M_{2}}{r^{5}}(r-M), \\
& 0=-v^{\prime}-\frac{4}{r} v+\frac{6 M^{2}}{r^{3}} \dot{k}-\frac{6 M M_{1} M_{2}}{r^{5}}(r-M), \\
& 0=-M \dot{k}^{\prime}+v+\frac{2 M}{r} \dot{k}+\frac{6 M M_{1} M_{2}}{r^{3}} .
\end{aligned}
$$

Here, a prime denotes differentiation with respect to $r$. The system (7,8) can be re-expressed as a single second order equation. Introducing the dimensionless quantities $x=r / M$ and $\mu=M_{1} M_{2} / M^{2}$, this equation reads

$$
0=-v_{x x}-\frac{5}{x} v_{x}+\frac{6}{x^{3}} v+\frac{6 \mu}{x^{6}}(3 x+2) .
$$


Once we have solved this equation, the remaining amplitudes $\dot{k}$ and $\dot{v}$ are obtained from (6) and (7), respectively. A particular solution of (9) is given by

$$
v(x)=-\frac{2 \mu}{3}\left(1-\frac{2}{x}+\frac{3}{x^{2}}+\frac{3}{x^{3}}\right) .
$$

In order to find the solutions of the homogeneous equation, one performs the transformations $x=24 / z^{2}, v(x)=z^{4} u(z)$, which yields the Bessel differential equation

$$
0=z^{2} u_{z z}+z u_{z}-\left(16+z^{2}\right) u
$$

The solutions are a linear combination of the Bessel functions $I_{4}(z)$ and $K_{4}(z)$. Thus, the general solution to (9) is $v(x)=\mu \hat{v}(x)$, with

$$
\hat{v}(x)=-\frac{2}{3}\left(1-\frac{2}{x}+\frac{3}{x^{2}}+\frac{3}{x^{3}}+\frac{A_{1}}{x^{2}} K_{4}(\sqrt{24 / x})\right)+\frac{A_{2}}{x^{2}} I_{4}(\sqrt{24 / x}) .
$$

Choices for $A_{1}$ and $A_{2}$ are discussed in the following section.

\section{B. Initial data for the Teukolsky function}

The initial data for the metric and extrinsic curvature obtained above will be evolved using the KS type, penetrating Teukolsky equation. This equation was derived by Campanelli et al. [7] in order to study gravitational perturbations close to the horizon. The equation, along with the associated geometrical quantity, the Newman-Penrose complex null-tetrad is regular at the horizon, and therefore has the capability of evolving through it. Such an equation is ideal to study the effects of "excision" as used by full numerical, binary black hole collision codes, like MAYA [6]. It should be noted, that the penetrating Teukolsky equation is based on a rescaling of the transformed Kinnersley tertrad (to KS coordinates). This rescaling enables the tetrad to be regular at the horizon. Therefore, the penetrating Teukolsky equation is not just the original Teukolsky equation in KS coordinates."?.

To obtain initial data for the Teukolsky function, we use the formula developed by Campanelli et al. [8] in terms of the perturbative three metric and extrinsic curvature as shown below. $\psi_{4}$ is a Newman-Penrose scalar, that captures information about outgoing gravitational radiation, and it is related to the Teukolsky function $\psi$ as, $\psi=r^{4} \psi_{4}($ for a non spinning black hole).

$$
\begin{aligned}
\psi_{4}= & -\left[R_{i j k l}+2 K_{i[k} K_{l] j}\right]_{(1)} n^{i} m^{j} n^{k} m^{l}+8\left[K_{j[k, l]}+\Gamma_{j[k}^{p} K_{l] p}\right]_{(1)} n^{[0} m^{j]} n^{k} m^{l} \\
& -4\left[R_{j l}-K_{j p} K_{l}^{p}+K K_{j l}\right]_{(1)} n^{[0} m^{j]} n^{[0} m^{l]} \\
\partial_{t} \psi_{4}= & N_{(0)}^{\phi} \partial_{\phi}\left(\psi_{4}\right)-n^{i} m^{j} n^{k} m^{l}\left[\partial_{0} R_{i j k l}\right]_{(1)} \\
& +8 n^{[0} m^{j]} n^{k} m^{l}\left[\partial_{0} K_{j[k, l]}+\partial_{0} \Gamma_{j[k}^{p} K_{l] p}+\Gamma_{j[k}^{p} \partial_{0} K_{l] p}\right]_{(1)} \\
& -4 n^{[0} m^{j]} n^{[0} m^{l]}\left[\partial_{0} R_{j l}-2 K_{(l}^{p} \partial_{0} K_{j) p}-2 N_{(0)} K_{j p} K_{q}^{p} K_{l}^{q}\right. \\
& \left.+K_{j l} \partial_{0} K+K \partial_{0} K_{j l}\right]_{(1)} \\
& +2\left\{\psi_{4}\left(l_{i} \Delta-m_{i} \bar{\delta}\right) N^{i(0)}+\psi_{3}\left(n_{i} \bar{\delta}-\bar{m}_{i} \Delta\right) N^{i(0)}\right\},
\end{aligned}
$$

where

$$
\begin{aligned}
\psi_{3}= & -\left[R_{i j k l}+2 K_{i[k} K_{l] j}\right]_{(1)} l^{i} n^{j} \bar{m}^{k} n^{l}+4\left[K_{j[k, l]}+\Gamma_{j[k}^{p} K_{l] p}\right]_{(1)}\left(l^{[0} n^{j]} \bar{m}^{k} n^{l}-n^{[0} \bar{m}^{j]} l^{k} n^{l}\right) \\
& -2\left[R_{j l}-K_{j p} K_{l}^{p}+K K_{j l}\right]_{(1)}\left(l^{[0} n^{j]} \bar{m}^{0} n^{l}-l^{[0} n^{j]} n^{0} \bar{m}^{l}\right),
\end{aligned}
$$

\footnotetext{
${ }^{1}$ We recently learnt that Carsten Kollein at Albert Einstein Institute performed a perturbative evolution of this same family of initial data in his M.Sc. thesis (unpublished), using the Teukolsky formalism. However, the Teukolsky equation used by Carsten was the Boyer-Lindquist Teukolsky equation transformed to KS coordinates, and not the penetrating Teukolsky equation. We thank Jorge Pullin and Manuela Campanelli for providing us with a copy of Carsten's work.
} 


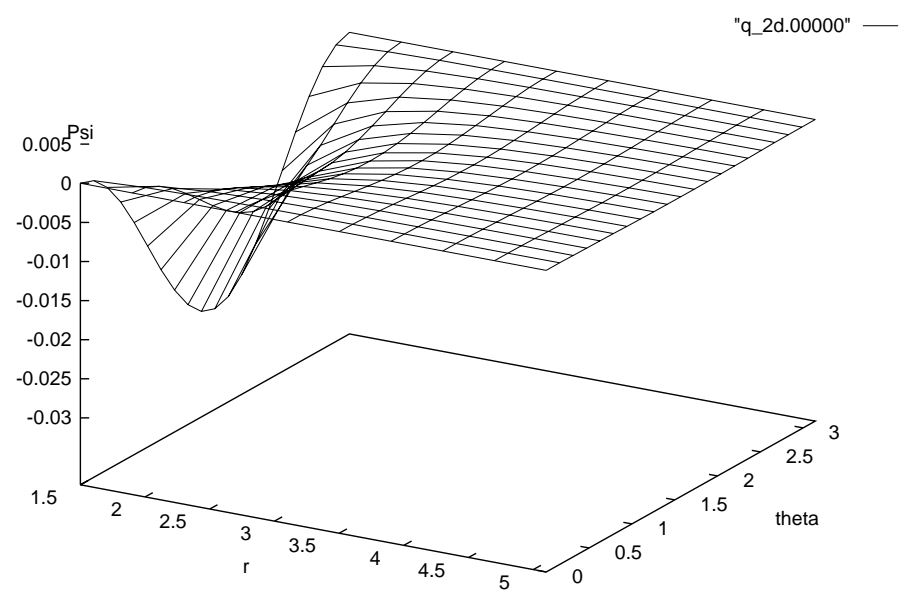

FIG. 1. The initial Teukolsky function, as function of $r$ and $\theta$. The value of the constant $A_{2}$ here is 0 .

$N_{(0)}=\left(g_{\text {kerr }}^{t t}\right)^{-1 / 2}$ is the zeroth order lapse, $n^{i}$ in these equations should be taken to be related to that of the original penetrating tetrad as $n^{0}=N_{(0)} n_{\text {orig }}^{0}, n^{i}=n_{\text {orig }}^{i}+N^{i}{ }^{(0)} n^{0}$. Latin indices run from 1 to 3 , and the brackets are computed to only first order (zeroth order excluded). The derivatives involved in the above expressions can be computed in terms of the initial data on the Cauchy hypersurface as,

$$
\begin{gathered}
\partial_{0} K=N_{(0)} K_{p q} K^{p q}-\nabla^{2} N_{(0)}, \\
\partial_{0} R=2 K^{p q} \partial_{0} K_{p q}+4 N_{(0)} K_{p q} K_{s}^{p} K^{s q}-2 K \partial_{0} K, \\
\partial_{0} R_{i j k l}=-4 N_{(0)}\left\{K_{i[k} R_{l] j}-K_{j[k} R_{l] i}-\frac{1}{2} R\left(K_{i[k} g_{l] j}-K_{j[k} g_{l] i}\right)\right\} \\
+2 g_{i[k} \partial_{0} R_{l] j}-2 g_{j[k} \partial_{0} R_{l] i}-g_{i[k} g_{l] j} \partial_{0} R+2 K_{i[k} \partial_{0} K_{l] j}-2 K_{j[k} \partial_{0} K_{l] i},
\end{gathered}
$$

and,

$$
\partial_{0} K_{i j}=N_{(0)}\left[\bar{R}_{i j}+K K_{i j}-2 K_{i p} K^{p}{ }_{j}-N_{(0)}^{-1} \bar{\nabla}_{i} \bar{\nabla}_{j} N_{(0)}\right]_{(1)} .
$$

We calculate the Teukolsky function $\psi$ and its time derivative $\dot{\psi}$, on the initial time slice, using the above formulae on expressions for the metric and extrinsic curvature that we produced before. The algebraic expressions for the initial data of the Teukolsky function are too large to include in this paper. Instead, we include some surface plots.

We still need to discuss the choices for the unknown constants $A_{1}$ and $A_{2}$ that appear in equation 10 and therefore in expressions for $\psi$ and $\dot{\psi}$. It turns out that in order for the Teukolsky function to have a proper fall-off behavior for large $r, A_{1}$ must equal -12 . This choice for $A_{1}$ also ensures that $v(r)$ vanishes at spatial infinity. $A_{2}$ on the other hand can be chosen at will. In their paper, Sarbach et al. provide a very clear explanation regarding the meaning of $A_{2}$ based on the original assertion by Bishop et al. They show that the meaning of $A_{2}$ is related to the position of the apparent horizon, through a genuine choice (not just a gauge choice) in the initial data.

In what follows, we shall choose $A_{1}=-12$ and use multiple values of $A_{2}$. In particular, we shall use $A_{2}=0$ and $A_{2}=10$ for our results.

\section{EVOLUTION}

Like we mentioned in the previous section, the initial data for the Teukolsky function obtained above, shall be evolved using the penetrating Teukolsky equation. This equation is best suited for comparisons with current KS type 


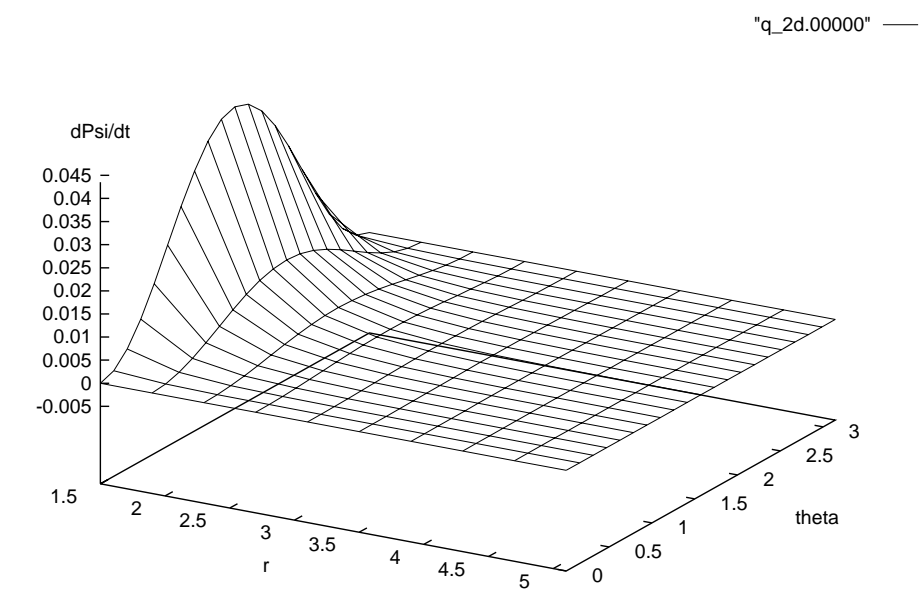

FIG. 2. The initial time derivative of the Teukolsky function, as function of $r$ and $\theta$. The value of the constant $A_{2}$ here is 0 .

full numerical binary black hole codes like MAYA, because its coefficients are regular at the horizon. This equation was derived by Campanelli et al. [7], and we reproduce it here (with the Kerr parameter, $a=0$ ),

$$
\begin{aligned}
& (\Sigma+2 M r) \frac{\partial^{2} \psi}{\partial t^{2}}-\triangle \frac{\partial^{2} \psi}{\partial r^{2}}-6(r-M) \frac{\partial \psi}{\partial r} \\
& -\frac{1}{\sin \vartheta} \frac{\partial}{\partial \vartheta}\left(\sin \vartheta \frac{\partial \psi}{\partial \vartheta}\right)-\frac{1}{\sin ^{2} \vartheta} \frac{\partial^{2} \psi}{\partial \varphi^{2}}-4 M r \frac{\partial^{2} \psi}{\partial t \partial r} \\
& +\left(\frac{4 i \cot \vartheta}{\sin \vartheta}\right) \frac{\partial \psi}{\partial \varphi} \\
& -(4 r+6 M) \frac{\partial \psi}{\partial t}+2\left(2 \cot ^{2} \vartheta-1\right) \psi=0 .
\end{aligned}
$$

To implement the penetrating Teukolsky equation numerically, we reduced it down into a $2+1$ dimensional one, using a decomposition of the Teukolsky function into angular modes, $\psi=\Sigma \psi_{m} e^{i m \varphi}$. We used Lax-Wendroff technique to numerically implement these simplified set of equations.

Results of evolutions for two equal mass KS holes are shown in the included figures. The inner boundary for the evolution was chosen to be inside the horizon, at $1.5 \mathrm{M}$. In figures 3 and 1 we show the Teukolsky function as a function of time, extracted at $r=50 \mathrm{M}$. We show waveforms for two values of $A_{2}$. Quasi-normal ringing is self-evident in these figures as expected. We also calculated the energy radiated for these evolutions, and we get amounts in agreement with those obtained by Sarbach et al.

$$
E=\varepsilon^{4} M\left(1.31 \times 10^{-4}+1.22 \times 10^{-6} A_{2}^{2}-1.92 \times 10^{-5} A_{2}\right)
$$

If we pick $A_{2}$ such that the energy radiated is at a mininum (this occurs at $A_{2}=7.87$ ), and we pick the conformally flat separation between the holes to be about $0.9 \mathrm{M}$, the value we obtain is about $10^{-5} \mathrm{M}$, which is consistent with the value obtained from the evolution of Misner data (with the same separation $L=0.9 M$ or $\mu_{0}=1.5$ ) using the original Teukolsky or Zerilli frameworks [9].

\section{CONCLUSIONS}

We evolved the initial data family of Bishop et al. in the close-limit by treating the spacetime as a single distorted Kerr-Schild black hole. We performed the evolution using the penetrating Teukolsky equation.

These results shall be used in the calibration of numerical codes based on the Kerr-Schild coordinates, e.g. MAYA [6]. They are also in agreement with those obtained by Sarbach et al. from their evolution of the same initial data family using a KS type Zerilli equation. 


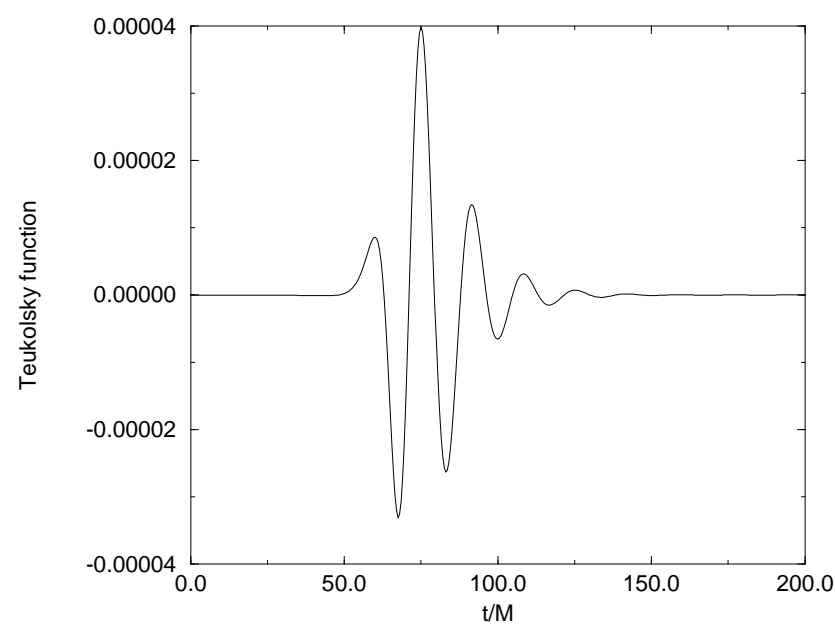

FIG. 3. The radiated waveforms at $r=50 M$ for $A_{2}=0$. As usual in close limit collisions, the waveform is dominated by the fundamental quasi-normal mode.

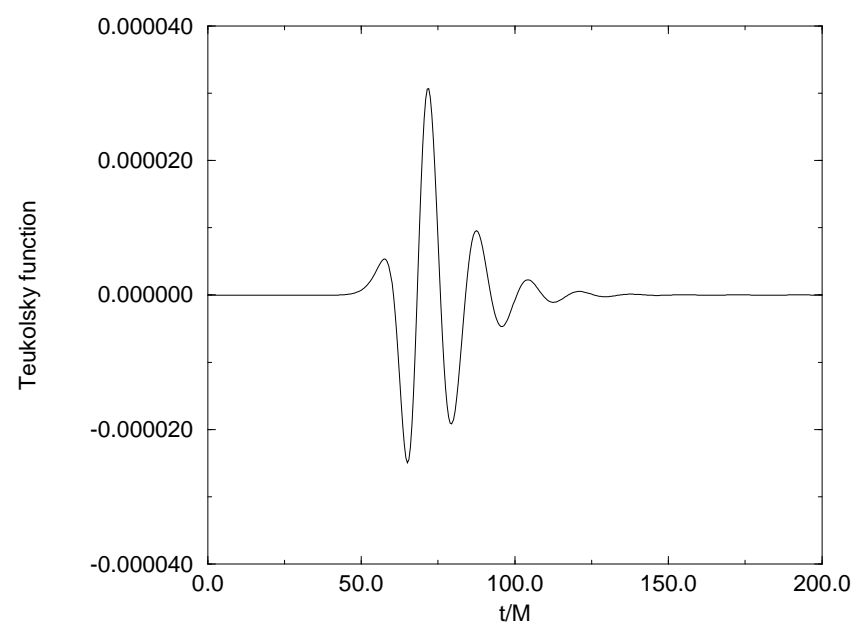

FIG. 4. The radiated waveforms at $r=50 M$ for $A_{2}=10$. As usual in close limit collisions, the waveform is dominated by the fundamental quasi-normal mode. 


\section{ACKNOWLEDGEMENTS}

We thank Long Island University for research support and computational facilities. We also thank Ashish Tiwari for helping out with some of the computational aspects of this paper, as well as proof reading it.

[1] G. Cook, Living Rev. Rel. 3, 5 (2000).

[2] N.T. Bishop, R. Isaacson, M. Maharaj, and J. Winicour, Phys. Rev. D57, 6113 (1998).

[3] O. Sarbach, M. Tiglio and J. Pullin, gr-qc/0110085.

[4] O. Sarbach and M. Tiglio, Phys. Rev. D64, 084016 (2001).

[5] G. Khanna, S. Liebling, work in progress.

[6] http://www.astro.psu.edu/nr/

[7] M. Campanelli, G. Khanna, P. Laguna, J. Pullin, M. P. Ryan, Class. Quant. Grav. 18 (2001) 1543-1554.

[8] M. Campanelli, C. O. Lousto, J. Baker, G. Khanna and J. Pullin, Phys. Rev. D58, 084019 (1998);

M. Campanelli, C. O. Lousto, J. Baker, G. Khanna and J. Pullin, Phys. Rev. D62, 069901(E), (2000).

[9] R. Price and J. Pullin, Phys. Rev. Lett. 72, 3297 (1994). 